\title{
Energy and Connectivity Performance of Routing Groups in Multi-radio Multi-hop Networks
}

\author{
Michele Rossi`, Leonardo Badia, Paolo Giacon, Michele Zorzi
}

\begin{abstract}
This paper explores the logical device aggregation of terminals in future generation networks, where the availability of several different radio access techniques is integrated by means of common radio resource management algorithms. In particular, we investigate the creation of routing groups among adjacent nodes, which might be beneficial in order to improve connectivity, decrease signaling overhead and increase transmission efficiency. A simple analytical approach is proposed, which allows the performance evaluation of device aggregation algorithms. We measure the performance of establishing routing groups with special focus on two metrics of interest: the connectivity of the nodes and the energy consumption. Within this framework, many detailed insights are obtained and presented throughout the paper. In particular, we focus on the effectiveness of these aggregation techniques in improving network connectivity and on the cost incurred in getting the extra information needed to build and maintain group structures. In the final part of the paper, we provide simulation results which further validate our discussion and highlight additional aspects that are to be considered in real scenarios. Our work is a first step in the investigation of the effectiveness of in-network aggregation of terminals equipped with multiple radio technologies. The results derived in the paper are encouraging and motivate further research on the topic.
\end{abstract}

keywords: routing groups, multiple radio technologies, radio technology diversity, radio access techniques, mobility exploitation, radio resource management, connectivity performance, analytical evaluation.

\section{INTRODUCTION}

Coexistence and integration of multiple access techniques (due to either coexisting multiple radio technologies or different service providers) over heterogeneous networks are a key issue for current research in wireless networks. The tremendous advancements achieved in the last few years in the wireless technology field have made it possible to integrate different radios in a single portable device, thereby opening up new marketing opportunities as well as new technological solutions. These themes are currently investigated under several international projects, among which we cite here the European Ambient Networks project [1].

\footnotetext{
${ }^{\star}$ Corresponding author, E-mail: rossi@ dei.unipd.it. M. Rossi and M. Zorzi are with the Department of Information Engineering, University of Padova, via Gradenigo 6/B, 35131 Padova, Italy and with the Consorzio Ferrara Ricerche (CFR), via Saragat 1, 44100 Ferrara, Italy. L. Badia is with the IMT Lucca Institute for Advanced Studies, via San Micheletto 3, 55100 Lucca, Italy. P. Giacon is with the Department of Innovation in Mechanics and Management, University of Padova, via Venezia 1, 35100 Padova, Italy. Part of this work has been presented at IEEE MobiWac 2005 (Best Paper Award).
}

In general, integration of multiple radio interfaces in the same device poses novel challenges, for both network operators and protocol designers. It becomes necessary, in fact, to provision efficient mechanisms to let such complex networks cooperate and possibly promote device aggregation and resource distribution in an efficient manner, so as to take advantage of the multi-radio technology diversity [2]. For instance, a possible problem to solve is how to connect every user to the "best" technology within range, at any time. In fact, the presence of multiple technologies has the potential to allow for increased performance as the system coverage, and hence the terminal connectivity, may be extended with respect to the single technology case. Furthermore, devices may decide, in an either coordinated or completely uncoordinated fashion, to switch to less congested systems, thereby achieving load balancing with a subsequent benefit in terms of user perceived performance and overall network utilization. However, these are just examples of the many issues that are to be solved in such networks.

In this paper, we mainly focus on the connectivity issue, where we are interested in understanding whether it is worth to perform logical device aggregation, also called "grouping." This is, in general, a theme addressed in past studies and often treated as an appropriate graph partitioning problem. Previous approaches are given in [3-5], where the clustering of network devices was used to improve routing as well as Medium Access Control (MAC) [6].

However, all these contributions focused on a single technology environment. The contribution of the present paper is considerably different as we add a new and important dimension to the device aggregation. In fact, we allow different technologies to coexist at both access points (APs) and devices. Moreover, we do not directly investigate strategies for realizing the terminals' aggregation, rather we seek an analytical evaluation of the impact of the routing group (RG) formation on two important metrics such as connectivity and energy consumption. As we will see in the following, the grouping concept allows to establish a trade-off between them, leading to generally improved performance at the price of a slight increase of the energy consumption. However, we argue that for realistic parameter settings this might be a good choice.

Our approach is quite general, since we are interested in a distributed topology network where aggregation of nodes is performed. Our study stems from the practical observation that mobile users often tend to move together, i.e., according to the so called group mobility behaviors $[7,8]$. Examples of group mobility might be found in our daily life, e.g., in a group of people in the same vehicle (car, shuttle, train, etc.) or pursuing a common task within the same geographical area (rescue squads, groups of tourists moving within a museum, etc.). In these cases, it might be beneficial for the users to perform logi- 
cal device aggregation and to elect leaders who are in charge of coordinating the transmissions within each group. Grouping, in some cases, may increase efficiency as well as connectivity of the terminals. For instance, the efficiency may be increased as the transmissions within every group may be handled locally by the group leader, thereby allowing for more efficient forwarding strategies. Our goal here is to derive an analytical model in order to capture the essential properties of this type of networks and to assess the possible benefits of device aggregation.

The remainder of this paper is organized as follows. First of all, in Section 2 we discuss the routing group concept as a means to harmonize mobile networks where different radio access technologies are present. In Section 3 we present the models that we propose to represent physical/transmission aspects such as user positions, radio interface distributions, propagation model and transmission powers. In Section 4, we characterize the RG size as a function of various system parameters. Such a characterization is the foundation for all the following analytical derivations. In Section 5, we briefly describe how algorithms for routing groups operate and we subsequently find the average energy spent to maintain RG structures. In Section 6, we focus on the analysis of the energy required to transmit to all users in the network with and without grouping. Based on our analytical framework, in Section 7 we present some results that highlight the benefit of grouping users in terms of improved connectivity of the terminals. In Section 8 we report preliminary but accurate simulation results to further confirm our discussion and highlight additional facts that would arise in real scenarios, i.e., by accounting for the highest level of detail (interference, time-variant channels and so on). Finally, in Section 9 we report the conclusions of our work.

\section{Heterogeneous Networks AND Routing GROUPS}

In this work, we address heterogeneous networks where users and APs possess multiple radio interfaces and operate within the same geographical area. In such an environment, it might be beneficial to join all or part of the users in what we refer to here as routing groups (RGs). This logical grouping is performed with the aim of taking advantage of the users' physical proximity and possibly of similar mobility patterns in order to improve the efficiency in transmitting data and/or handling network related procedures such as the handover between different APs. As an example, multiple users moving together and handing over at the same time between the same pair of APs may be joined in a routing group so that a single message (to the RG leader) needs to be exchanged to successfully accomplish the handover procedure, instead of using one dedicated channel (a unicast message) for every user. In general, this is true every time the transmission involves information content that can be shared among users, that is, for all applications where some sort of multicast messaging is inherently supported. In other cases, we may join users according to their access technologies and "cluster" them to increase the transmission efficiency. Think again, for instance, of a vehicle occupied by several passengers, which henceforth move with the same pattern. In such a case, it could be efficient to elect a RG leader, which is typically chosen among the most capable devices, e.g., the on-board multimedia system, and transmit the information related to, e.g., close tourist attractions, route information, TV programs, to all users in the vehicle in a multicast fashion. In such a case, the RG leader will retrieve the wanted information from the external network through dedicated access points, and then the information could be more efficiently distributed to the RG members by exploiting their physical proximity. This simple example illustrates the opportunities and advantages offered by a grouping of network entities when they exhibit a group mobility behavior.

In this work, instead of deriving specific algorithms for handling and creating RGs, we focus on the effectiveness of the grouping principle as a function of the node and RG leaders densities, and of the number and type of radio interfaces owned by the users. In particular, our aim is to quantify such benefits and weigh them against the costs incurred in creating and maintaining RG structures.

\section{System MOdeL}

We consider a heterogeneous network where a number of access points (APs) and a number of users coexist. Both APs and users support multiple radio technologies which can be described by the indices $1,2, \ldots, J$. We assume the knowledge of three vectors $\mathbf{E}^{t x}=\left\{E_{1}^{t x}, E_{2}^{t x}, \ldots, E_{J}^{t x}\right\}, \mathbf{E}^{r x}=$ $\left\{E_{1}^{r x}, E_{2}^{r x}, \ldots, E_{J}^{r x}\right\}$ and $\mathbf{r}=\left\{r_{1}, r_{2}, \ldots, r_{J}\right\}$ tracking the energies required to transmit and receive a single bit and the transmission ranges for every technology, respectively. In the following, we assume that indices are sorted according to the transmission range of the related interface, i.e., $1 \leq h<j \leq$ $J \Leftrightarrow r_{h} \leq r_{j}$ (if $r_{h}=r_{j}$ their order is irrelevant). These assumptions mean that, for the sake of simplicity, we do not investigate Power Control issues, even though we add some considerations in the following. We also simplify the MAC by considering that different transmissions (of different terminals or on different interfaces) might occur simultaneously without causing interference or collisions. This can be done, since we are mainly interested in estimating the connectivity issue and the overall energy consumption (and not, for example, in the error probability of received transmissions), so that even a simplified radio model is able to give useful insight. It is obviously possible to replace these assumptions with more complicated and detailed descriptions of the MAC, but this would be beyond the scope of the present paper. However, we argue that this can be done by following the same rationale we present in the following. Moreover, in what follows we discuss possible ways of connecting our scenario (with simplified assumptions due to the analytical approach) with realistic physical parameters and propagation aspects.

APs are assumed to possess all the available technologies and are therefore able to communicate with every device within range. Instead, not all nodes offer all radio interfaces and, in general, the set of available interfaces may differ between different nodes. In order to have an easily tractable analytical model, we simply assume that every node owns the $j$ th interface of the network with probability $p_{j}$. Observe that in our model the $p_{j}$ s do not sum to one as they do not represent a probability mass function over the possible interfaces. For a given $j, p_{j}$ is constant for all nodes and the probability of the presence of any interface at a given node is independent of the presence of other interfaces. This might lead to the possibility of nodes without any interface, which describes the case 
of terminals without a compatible interface with the core network (i.e., the set of the APs). Besides, this assumption must be seen mainly as a mathematical hypothesis made for analytical simplicity, which can be removed at the price of obtaining more cumbersome expressions.

For the topology, both users and APs are placed according to planar Poisson processes of density $\rho$ and $\rho_{A P}$, respectively. That is, the number of nodes in a given area follows a Poisson probability density function (pdf), whereas conditioned on the number of devices, node positions are uniformly distributed within the area [9]. At the physical layer, every transceiver device has a given receiver sensitivity $\eta_{j}$ which depends on the considered radio interface $j \in\{1,2, \ldots, J\}$. We assume that packets can be correctly decoded when the received power is above the respective technology-dependent sensitivity threshold. The propagation loss $L(d)$ (in decibel) at a distance $d$ can be modeled as $L(d)=K_{0}+K_{1} \ln d+s$, where $K_{0}$ and $K_{1}$ are proper constants, and $s$ is a shadowing sample which is assumed to be normally distributed with zero mean and standard deviation $\sigma_{\text {shad }}$. Thus, the received power (decibel) at the generic interface $j$ of a given node is $P_{r x, j}(d)=P_{t x, j}-L(d)$, where $d$ is the distance between the source (S) and the node itself and $P_{t x, j}$ is the power used by $\mathrm{S}$ to transmit. We say that a packet transmitted with technology $j$ is correctly received if $P_{r x, j}(d) \geq \eta_{j}$. Observe that, as the channel attenuation is modeled accounting for a log-normal shadowing contribution, the received power (and hence the correctness of a packet transmission) as a function of the distance is a random variable depending on the shadowing pdf. Now, if we refer to a probabilistic threshold $p_{c} \in(0,1)$, we can define the maximum transmission range $r_{j}$ for a given technology $j \in\{1,2, \ldots, J\}$ as the maximum distance $d_{\max , j}$ for which $\operatorname{Prob}\left\{P_{r x, j}(d) \geq \eta_{j}\right\}=p_{c}$. Then, we can set $r_{j}=d_{m a x, j}$ by observing that $r_{j}$ is conditioned on the quality of service (QoS) level captured by the probability $p_{c}$. Hence, by repeating the above reasonings for every technology, it is possible to derive the maximum transmission range vector $\mathbf{r}$ as a function of the transmission power levels $P_{t x, j}$ and of the radio sensitivities $\eta_{j}$, where $\mathbf{r}$ is conditioned on the minimum QoS guarantee $p_{c}$, as explained above. That is, given the QoS requirements, we can always obtain the corresponding vector of maximum transmission distances. Given the network topology and the radio interface models, the density $\rho_{j}$ of nodes with an interface of type $j$ is $\rho_{j}=p_{j} \rho$. Note that, $\sum_{j=1}^{J} \rho_{j}$ may be larger than $\rho$.

In Fig. 1, we report an illustrative example of the considered network architecture. Both nodes and APs are randomly and uniformly placed over the area. Network devices are classified in two different categories: regular devices (referred to as $D e-$ vice in the figure) and routing group leaders (referred to as $R G$ leaders). Like the APs, RG leaders are also assumed to have all technologies, whereas regular devices own any technology $j \in\{1,2, \ldots, J\}$ with probability $p_{j}$. As reported in the figure, we account for two different communication paradigms: in the first case (e.g. AP3) nodes communicate directly with the closest AP, whereas in the second case (e.g. AP2 in the figure) nodes communicate with their RG leader which acts as a relay node for every device in its RG. The aim of the following analysis is to compare these two possibilities in terms of energy consumption as well as network connectivity.

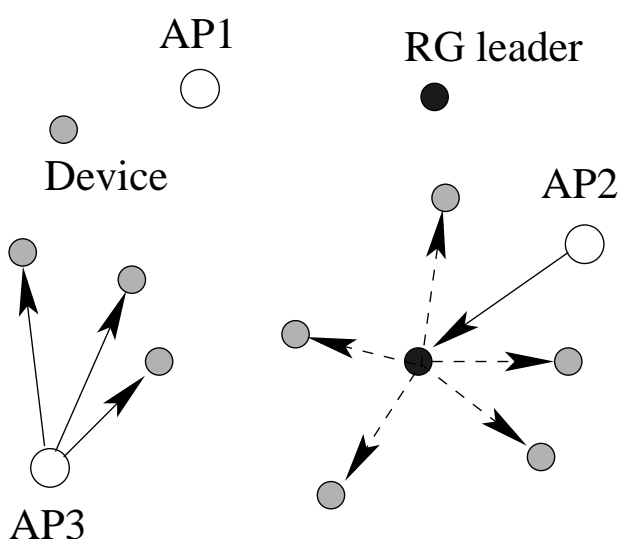

Fig. 1. Considered network architecture.

\section{Calculation of the Average Routing Group Size AND NUMbER OF MEMbERS}

In this section we characterize the RG structure, by analyzing the RG geographical extension and number of nodes. These results will be used in the following sections for the calculation of the average energy required to deliver data when RGs structures are in place. For the sake of simplicity, we consider that RG leaders are uniformly distributed within the network and that the value $p_{L}$ represents the probability that an arbitrarily picked node is a leader [10]. RG leaders are elected first according to the probability $p_{L}$ and are assigned all available technologies. On the other hand, all other nodes which are not RG leaders are assumed to own technology $j=\{1,2, \ldots, J\}$ with probability $p_{j}$, as explained in the previous section. This approach describes in an exact manner any leader selection strategy based on random election and also approximates reasonably well other strategies. In fact, if needed, it can be replaced by a more refined procedure which also accounts, e.g., for the correlation of leader positions when computing inter-leader distances (so that, for example, neighborhood among leaders is less frequent), by replacing $p_{L}$ in the following Eq. (3) with a probability depending on the number of hops separating two adjoining RG leaders.

We note that depending on the specific RG scheme at play, the average RG size may vary, as for standard clustering approaches $[11,12]$. Hence, we can choose $p_{L}$ to reflect, in a very simple manner, the average size of the formed RGs and hence to account for the specific RG formation algorithm. For what concerns the node positioning, we still consider all devices (standard nodes and RG leaders) to be placed according to a Poisson distribution. Now, we focus on a given node and we assume that the node was elected as a RG leader. Then, starting from this leader, we seek for the 1 st, 2 nd, $\ldots, n$th device surrounding it, where the 1 st node is the closest to the leader, the 2nd is the second closest and so on. Moreover, we refer to $d_{1}, d_{2}, \ldots, d_{n}$ as the random values of the distances between the leader and these $n$ nodes. The joint pdf of the $d_{j}$ 's was first derived in [13] and is given by

$$
\psi\left(d_{1}, d_{2}, \ldots, d_{n} \mid n\right)=(2 \lambda)^{n} e^{-\lambda d_{n}^{2}} d_{1} d_{2} \cdots d_{n},
$$

where $\lambda=\pi \rho$ and $0 \leq d_{1} \leq d_{2} \leq \cdots \leq d_{n}$. The absolute probability that the $n$th nearest neighbor is distant $\tilde{d}$ from the RG leader is obtained by integrating Eq. (1) with respect to $d_{1}$ 
from 0 to $d_{2}$, with respect to $d_{2}$ from 0 to $d_{3}, \ldots$, with respect to $d_{n-1}$ from 0 to $d_{n}=\tilde{d}$ and is given by:

$$
P(\tilde{d})=\frac{2 \lambda^{n} e^{-\lambda \tilde{d}^{2}} \tilde{d}^{2 n-1}}{(n-1) !} .
$$

Moreover, the probability that the closest leader is the $n$th nearest node is given by:

$$
P\{\text { node } n \text { is the closest leader }\}=\left(1-p_{L}\right)^{n-1} p_{L} .
$$

The joint pdf that the $n$-th closest node is the closest leader and its position is $\tilde{d}$ is then given by the product of Eqs. (2) and (3) as follows:

$$
\begin{aligned}
& P\{n \text {th neighbor is the closest leader, } \tilde{d}\} \\
& =\frac{2 \lambda^{n} e^{-\lambda \tilde{d}^{2}} \tilde{d}^{2 n-1}\left(1-p_{L}\right)^{n-1} p_{L}}{(n-1) !} .
\end{aligned}
$$

The marginal pdf $\psi(\tilde{d})$ is therefore found as:

$$
\begin{aligned}
& \psi(\tilde{d})=\sum_{n=1}^{\infty} P\{n \text {th neighbor is the closest leader, } \tilde{d}\} \\
& =\beta \sum_{n=1}^{\infty} \frac{\alpha^{n}}{(n-1) !}=\beta \alpha e^{\alpha}
\end{aligned}
$$

where $\alpha=\left(1-p_{L}\right) \lambda \tilde{d}^{2}$ and $\beta=\left[p_{L} /\left(1-p_{L}\right)\right] \tilde{d} e^{-\lambda \tilde{d}^{2}}$, so that $\psi(\tilde{d})$ can be re-written as:

$$
\psi(\tilde{d})=2 \lambda p_{L} \tilde{d} e^{-\lambda p_{L} \tilde{d}^{2}} .
$$

Now, the average closest distance between two leaders can be computed by:

$$
\mathbb{E}[\tilde{d}]=2 \gamma \int_{0}^{\infty} x^{2} e^{-\gamma x^{2}} \mathrm{~d} x=\frac{1}{2 \sqrt{\rho p_{L}}},
$$

where $\mathbb{E}[\tilde{d}]$ indicates the expectation of $\tilde{d}$ and $\gamma=\lambda p_{L}$. This result, obtained for uniform node distribution, can be extended by following a similar approach to more complicated cases. In fact, as shown in [14], analytical results are available to quantify the error introduced by using a Poisson approximation when the underlying process of the node distribution is not stationary. From Eq. (7), we can calculate the average range $\left(\bar{r}_{R G}\right)$ as $\mathbb{E}[\tilde{d}] / 2$. In other words, $\pi r_{R G}^{2}$ is the average area served by a RG leader. Therefore:

$$
\bar{r}_{R G}=\frac{1}{4 \sqrt{\rho p_{L}}} .
$$

According to the propagation model discussed in Section 3, in the following we assume that the propagation medium is characterized by circular coverage areas, so that the average area $\left(\mathcal{A}_{R G}\right)$ covered by a RG is determined as $\mathcal{A}_{R G}=\pi \bar{r}_{R G}^{2}$. For the specific case under exam, we claim that the restriction to the investigation of circular areas, apart from keeping the analysis simple, still has the merit of giving direct insight without limiting too much the validity of the approach. Real coverage areas are not exactly circular, due to border effects. Moreover, if one wanted to include more directly shadowing or Rayleigh fading [15] for each of the radio interfaces, the coverage area would be different and no longer circular.
For these cases, which are currently under study, it is possible to apply to some extent the general results found on clustering evaluation, in particular for what concerns the extension of the coverage areas from circular or analogously simple shapes to generalized regions. In this view, it has been shown [16] that a Poisson approximation allows to follow the same rationale that we will develop in the next sections, with known results about the introduced approximation. For these reasons, we argue that our evaluations are general enough, since we only focus here on average values. In order to investigate the variation of the results instead, further research might be needed to deal with the case of generic coverage area, which introduces a further deviation. Also, we note that throughout the following analysis we will account for the area covered by a RG by means of the above Eq. (8). We observe that this consists of a first order approximation that, however, will not affect the validity of concepts discussed in the present paper. Besides, in Section 8 we will confirm our analysis by means of simulation results.

\section{RG FORMATION AlgorithmS AND RELATED ENERGY CONSUMPTION}

RGs can be usually formed exploiting a distributed approach. That is, users cooperate and exchange data in order to gain information about their physical proximity and, at the same time, to measure the worthiness of grouping with other network entities. In general, the creation of group structures within a network can be achieved by the periodical exchange of so called HELLO messages between nodes [17-19]. In our scenario, things are complicated by physical mobility, so that the connectivity of a node might be subject to sudden changes. However, it is easy to understand that if movements are correlated, certain nodes are likely to remain in close proximity of the sending device and are therefore good candidates to be grouped with it. We therefore assume that the aggregation algorithm is able not only to detect the reachability of a neighboring node, but also to give an estimate of the "stability" of a connection, i.e., its likelihood of being available in the future, so that we might focus only on stable neighbors. Albeit specific algorithms for the creation of these RG structures are not in the scope of the present paper, we simply observe here that this stability can be evaluated by appropriate exchange of signaling information. For example, the nodes might include in each HELLO the list of their stable neighbors, which might be initialized as the list of nodes that have been in close proximity for a long enough period of time [8]. Additionally, this measurement might be reinforced by comparison of data coming from different neighbors, so that the initial estimate provides an accurate enough evaluation of a routing group which keeps stability in the near future [19]. We refer the interested reader to [19] for practical algorithms for the creation of RG structures in a distributed fashion by accounting for physical layer and MAC issues.

We further assume that a leader is elected within each RG. This device has the special role of handling the data traffic so as to optimize the transmission and the channel access of the RG members. This can be seen, as in standard clustering algorithms for ad hoc networks $[11,12]$, as a way to partially centralize the transmission control, thereby enhancing the performance. We assume that every interface $j \in\{1,2, \ldots, J\}$ 
sends HELLO messages with an interface-specific period $T_{j}$ and we refer to $b_{j}$ as the number of bits composing HELLO packets sent by an interface of type $j$. Moreover, we consider that all $T_{j}$ s are multiple of a reference time period $\Delta T$ such that $T_{j}=\xi_{j} \Delta T, \xi_{j} \in \mathbb{Z}^{+}, j \in\{1,2, \ldots, J\}, \Delta T \in \mathbb{R}^{+}$. If we define the least common multiple (LCM) of all $\xi_{j} \mathrm{~s}$ as $\xi$, then we have that:

$$
H_{j}=\frac{\xi}{\xi_{j}}
$$

is the number of HELLOs sent by the $j$-th interface in a time period equal to $\xi \Delta T$. According to the above model and assumptions, the energy spent to maintain the RG structures over an area $\mathcal{A}$ in a time period of $\xi \Delta T$ seconds can be well approximated as:

$$
\begin{gathered}
\bar{E}_{(m) R G}(\mathcal{A}, \xi \Delta T)=\sum_{j=1}^{J} \sum_{n=1}^{\infty} \mathcal{P}(n, \mathcal{A}) \\
\cdot \sum_{k=1}^{n}\left\{k P_{j}(k \mid n) b_{j} H_{j}\left[E_{j}^{t x}+E_{j}^{r x} \varepsilon_{j}\right]\right\} \\
\varepsilon_{j}=\sum_{n=2}^{\infty} \mathcal{P}\left(n, \pi \min \left(r_{j}, \bar{r}_{R G}\right)^{2}\right) \sum_{k=1}^{n}(k-1) P_{j}(k \mid n),
\end{gathered}
$$

where $P_{j}(k \mid n)=\left(\begin{array}{l}n \\ k\end{array}\right) p_{j}^{k}\left(1-p_{j}\right)^{n-k}$. In the above Eq. (10), the term $k H_{j}$ gives the number of HELLOs sent for an interface of type $j$ in a time frame of $\xi \Delta T$ seconds, given that there are exactly $k$ nodes within the area $\mathcal{A}$ owning such an interface. $k b_{j} H_{j}\left[E_{j}^{t x}+E_{j}^{r x} \varepsilon_{j}\right]$ accounts for the energy spent in sending those packets. Further, this last term is averaged according to the probability of having $k$ nodes out of $n$ with interface of type $j\left(P_{j}(k \mid n)\right)$. In addition, we take a double expectation over the interface set $(j=1,2, \ldots, J)$ and the number of nodes $n$ in $\mathcal{A}$. Finally, $\varepsilon_{j}$ is the mean number of devices receiving the HELLO message sent by a given sending node and using interface $j$; this term is accounted for to reflect the energy spent in receiving HELLO messages. In its calculation, we reasonably assume that HELLO packets are only decoded by the node neighbors whose distance is less than or equal to $\bar{r}_{R G}$, i.e., in the worst case RG related information spans over two adjoining RGs. ${ }^{1}$ The energy spent per unit of area and time to create and maintain RG structures is therefore derived as:

$$
\bar{E}_{(m) R G}^{*}=\frac{\bar{E}_{(m) R G}(\mathcal{A}, \xi \Delta T)}{\mathcal{A} \xi \Delta T} .
$$

This calculation holds for a uniform node and radio interface distribution and for a generic RG grouping algorithms where RGs are formed and maintained in a distributed manner thanks to periodic exchanges of neighborhood information. Moreover, Eq. (10) is related to the maintenance phase, whereas the initial transient (discovery) phase, which could be reasonably characterized by a higher energy consumption is neglected as it does not contribute to the steady-state energy metric.

\footnotetext{
${ }^{1}$ Devices may decide, based on the RG membership information contained into the HELLO packet header, whether they should decode or ignore the packet (thereby saving energy). This mechanism could be implemented through special header tags as done, e.g., in the Bluetooth system [20] to discriminate packets belonging to different piconets.
}

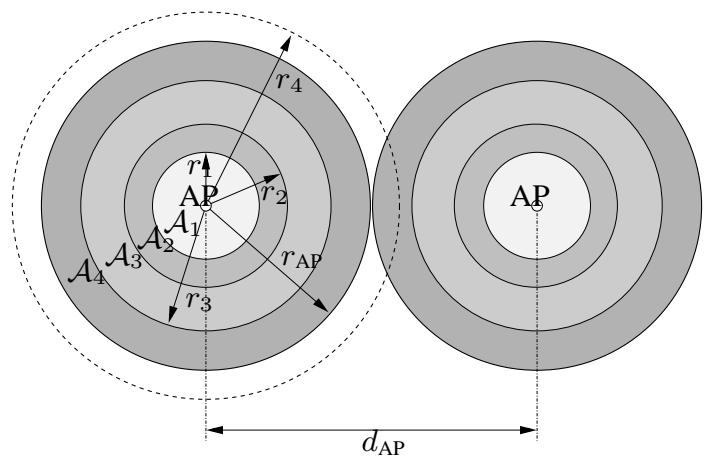

Fig. 2. Illustration of the AP coverage capabilities and their relation to the radio technologies transmission ranges.

In the following sections, we consider the data transmission by focusing on the scenarios with and without RGs. Observe that, in the former case flows are routed first from the closest AP to the RG leader (AP $\rightsquigarrow$ RG leader) and then optimally and locally distributed to the RG members (RG leader $\rightsquigarrow$ RG members). In the latter scenario (no RGs), instead, flows are transmitted directly by the APs to every device in the network. See Fig. 1 for an example of the above two cases, where the former is illustrated by AP2, whereas the latter is represented by the transmission originated from AP3.

\section{Connectivity AND EnERgy CONSUMPTION ANALYSIS}

In this section we consider the delivery of traffic to a set of users surrounding a given AP. We assume that each user requires a separate flow and all flows have the same bit-rate $B_{U}{ }^{2}$ These two assumptions can be seen as the situation where all nodes in the network are active and the common bit-rate can be roughly interpreted as the average transmission rate delivered to the end users. The aim of the following analysis is to characterize the energy spent per unit of area and time in transmitting these flows to all users in the network. We further consider that APs are placed according to a uniform distribution with density $\rho_{A P}$ and are equipped with all the technologies present in the network. The average distance between two APs is therefore given by $\bar{d}_{A P}=1 /\left(2 \sqrt{\rho_{A P}}\right)$ (Eq. (7) with $p_{L}=1$ ).

For the sake of simplicity, we assume that the area covered by every AP can be approximated by a circle. It is true that circular regions do not perfectly cover the plane. However, this still gives qualitatively correct results as it respects the quadratic proportionality between $\bar{d}_{A P}$ and the actual average area spanned by an AP. Moreover, to have a more accurate evaluation of how serving areas partition the plane, more information is needed than the average distance between APs, which is beyond the scope of the present paper. Accordingly, on average each AP is in charge of delivering data to all users placed within a circle of radius $\bar{r}_{A P}=\bar{d}_{A P} / 2$.

To help understand the following analysis, in Fig. 2 we report a scheme depicting two neighboring APs and the radio technologies transmission ranges (vector $\mathbf{r}$ ) in a scenario

\footnotetext{
${ }^{2}$ This assumption is made here to keep the analysis simple but it can be easily removed at the price of a further expectation, by following a similar approach.
} 
with $J=4$ different radio technologies. In normal situations, the larger the coverage, the higher the power expenditure. However, to have an approach as general as possible, we re-index the coverage regions according to the power consumption required to cover them. This is possible by defining an appropriate indexing (hence invertible) function $\mathfrak{i}(\cdot)$ from $\{1,2, \ldots, J\}$ to itself, so that the indices $1,2, \ldots, J$ are sorted as in Section 3 according to the coverage ranges, whereas $\mathfrak{i}(1), \mathfrak{i}(2), \ldots, \mathfrak{i}(J)$ rank the interfaces according to the power expenditure, i.e., $E_{h}^{t x} \leq E_{j}^{t x} \Leftrightarrow \mathfrak{i}(h)<\mathfrak{i}(j)$. It is important to note that in most cases the same order for power expenditure and coverage holds.

The area covered by the AP can be sliced into $J$ regions $\mathcal{A}_{1}, \mathcal{A}_{2}, \ldots, \mathcal{A}_{J}$, where the area $\mathcal{A}_{j}=0$ if $r_{j-1}>\bar{r}_{A P}$, otherwise the region has the shape of a circular annulus with area $\left.\pi\left[\min \left(\bar{r}_{A P}, r_{j}\right)^{2}-r_{j-1}\right)^{2}\right]$, where $r_{0}$ is 0 by definition.

The density of nodes with technology $j$ is still given by $\rho_{j}=\rho p_{j}$. The average number of users that have to be reached in the $j$ th region, $\bar{n}_{j}$, is found according to:

$$
\bar{n}_{j}=\rho \mathcal{A}_{j} .
$$

Note that if technology $J$ (the one with highest coverage) can not completely cover the serving area assigned to the AP (that is $r_{J}<\bar{r}_{A P}$ ), part of the area assigned to the AP, i.e., $\pi\left[\bar{r}_{A P}^{2}-\right.$ $\left.r_{J}^{2}\right]$, remains uncovered.

\section{Case without RGs}

The aim of this section is to compute the average energy required to deliver the flows to all users served by a given AP. We assume that the AP has a complete knowledge regarding the users to be served and can therefore optimize its transmission energies as follows. First of all, the AP serves all users in $\mathcal{A}_{1}$ having technology $\mathfrak{i}(1)$, hence $\bar{n}_{1, \mathfrak{i}(1)}=\rho_{\mathfrak{i}(1)} \mathcal{A}_{1}$ users are served (on average), where we refer to $\bar{n}_{j, h}$ as the average number of users served in region $j$ by technology $h$. For what concerns technology $i(2)$, the AP on average needs to serve $\bar{n}_{1, \mathfrak{i}(2)}=\bar{n}_{1}\{\mathfrak{i}(2)$ is opt $\}=\rho p_{\mathfrak{i}(2)}\left(1-p_{\mathfrak{i}(1)}\right) \mathcal{A}_{1}$ users in region 1 , where in general $\bar{n}_{j, h}=\bar{n}_{j}\{h$ is opt $\}$ is the average number of users in region $j$ for which interface $h$ is the optimal choice, that is, it is the least energy consuming interface among the ones at disposal and which can be reached by the AP. In general, in region $\mathcal{A}_{j}$, the probability of having $k$ users over $n \geq k$ with interface $h$ but without all interfaces $\ell$ which both cover $\mathcal{A}_{j}(\ell \geq j)$ and are less energy consuming than $h$ $(\mathfrak{i}(\ell)<\mathfrak{i}(h))$, is:

$$
\begin{aligned}
& P\{h \text { is opt in region } j\}(k \mid n)= \\
& \begin{cases}\left(\begin{array}{l}
n \\
k
\end{array}\right)\left(\tilde{p}_{h j}\right)^{k}\left(1-\tilde{p}_{h j}\right)^{n-k} & \text { if } h \geq j \\
0 & \text { if } h<j,\end{cases}
\end{aligned}
$$

where $\tilde{p}_{h j}=p_{h}\left[\prod_{\ell \geq j, \mathfrak{i}(\ell)<\mathfrak{i}(h)}\left(1-p_{\ell}\right)\right]$, and $p_{h}, p_{\ell}$ are the probabilities for a generic user of having interface of type $h$ and $\ell$, respectively.

The average number of users $\bar{n}_{j}\{h$ is opt $\}$ in region $j$ that can be optimally covered by exploiting interface $h$ is therefore found by averaging over the Poisson distribution, accounting for the probabilities $P\{h$ is opt in region $j\}(k \mid n)$ and finally

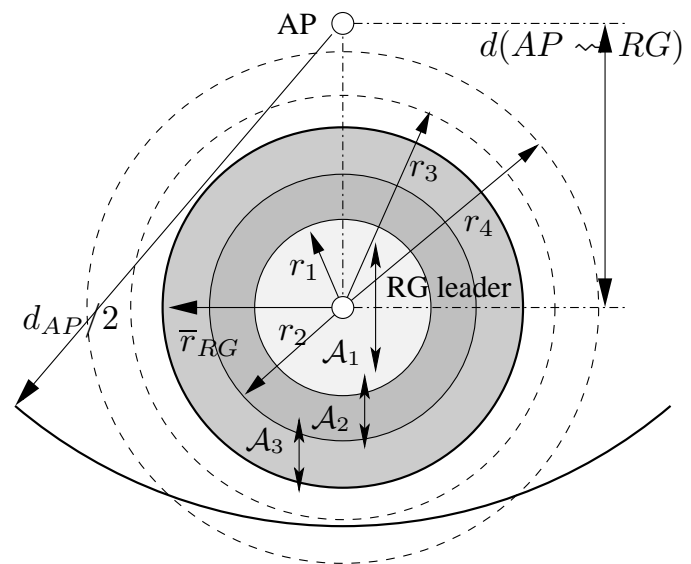

Fig. 3. Diagram for the calculation of the energy spent in transmitting unicast traffic in the RG case.

multiplying by the area $\mathcal{A}_{j}$. Hence:

$$
\bar{n}_{j, h}=\bar{n}_{j}\{h \text { is opt }\}= \begin{cases}\rho \tilde{p}_{h j} \mathcal{A}_{j} & h \geq j \\ 0 & h<j .\end{cases}
$$

The total energy expenditure per unit area and time is therefore found as:

$$
\bar{E}_{n o R G}=\frac{\sum_{h=1}^{J} \sum_{j=1}^{J} \bar{n}_{j, h}\left(E_{j}^{t x}+E_{j}^{r x}\right) B_{U}}{\pi \bar{r}_{A P}^{2}} .
$$

Besides the energy expenditure, another interesting performance metric to look at is the average number of uncovered users. These are users who can not be reached even by the closest AP with any of the radio interfaces they are equipped with. Their average number $\bar{n}_{u}$ is obtained by subtracting the value $\bar{n}_{j, h}$ summed over all technologies $h$ and regions $j$, from the average number of users which fall within $\bar{r}_{A P}$ meters from the AP. This leads to:

$$
\bar{n}_{u}=\pi \bar{r}_{A P}^{2} \rho-\sum_{j=1}^{J} \sum_{h=1}^{J} \bar{n}_{j, h} .
$$

In this way, we also account for completely uncovered regions (when present). The number of uncovered users per unit area is finally given by $\bar{n}_{u} /\left(\pi \bar{r}_{A P}^{2}\right)$.

\section{Case with RGs}

In this section we consider the scenario where the traffic has to be delivered to all users through dedicated channels, and RG structures are present in the network. In this case, instead of directly transmitting the data traffic to the end users we rely on the presence of RG leaders. As above, we consider a downlink transmission for each data flow, where all flows are assumed to have the same bit-rate $B_{U}$ and one data flow has to be delivered to each user. The diagram for this case is depicted in Fig. 3, where we report an example scenario with $J=4$ radio interfaces. According to the analysis in the previous section, we characterize the AP coverage radius by means of $\bar{r}_{A P}$, whereas the RG area covered by the RG leader is modeled through $\bar{r}_{R G}$, see Eq. (8). In this case, the unicast flows are first transmitted to the RG leader and then optimally delivered from here to the nodes in the RG coverage area. As per 
the aforementioned working assumption, we assume that RG leaders have all technologies as in practice it is reasonable to pick RG leaders among the more capable devices. As for the case without routing groups, we subdivide the RG area into $J$ regions (in Fig. 3, $J=4$ ) and we calculate the average number of reachable users in a RG as $\bar{n}_{R G}=\sum_{h=1}^{J} \sum_{j=1}^{J} \bar{n}_{j, h}$, where the quantities $\bar{n}_{j, h}$ are evaluated from the analysis illustrated in Section 6-A by substituting $\bar{r}_{A P}$ with $\bar{r}_{R G}$, see Eqs. (8) and (15). Note that $\bar{n}_{R G}$ is the average number of users served by a RG leader given that this leader actually exists. More details on this are given later in this section (see Eq. (21)).

In the RG case the transmission takes place in two different phases, where the first one consists of the transmission from the APs to the RG leaders (AP $\rightsquigarrow$ RG leader) and the second one of the transmission from the RG leaders to the RG members (RG leader $\rightsquigarrow R G$ members). Additionally, also the energy consumed to maintain the RG structure has to be taken into account. Thus, the overall energy expenditure per unit area and time, called $\bar{E}_{R G}^{*}$, where the asterisk indicates the normalization, can be subdivided into three different contributions, i.e., the energy required to transmit the flow from the AP to the RG leader, called $\bar{E}_{(a) R G}^{*}$, the further contribution required to deliver the flow from the leader to the nodes, $\bar{E}_{(b) R G}^{*}$, and finally the energy to maintain the RG structure $\bar{E}_{(m) R G}^{*}$. Formally:

$$
\bar{E}_{R G}^{*}=\bar{E}_{(a) R G}^{*}+\bar{E}_{(b) R G}^{*}+\bar{E}_{(m) R G}^{*} .
$$

The contribution $\bar{E}_{(m) R G}^{*}$ has been already determined by Eq. (12). The second term $\bar{E}_{(b) R G}^{*}$ can be seen as a specialization of the algorithm presented in Section 6-A where the RG size $\left(\bar{r}_{R G}\right)$ is used instead of the AP coverage area $\left(\bar{r}_{A P}\right)$.

For what concerns the first energy contribution $\bar{E}_{(a) R G}^{*}$, which involves the transmission (AP $\rightsquigarrow$ RG leader), we reasonably assume that RG sizes are significantly smaller than the AP coverage area, i.e., that $\bar{r}_{R G}^{2} \ll \bar{r}_{A P}^{2}$. In such a case, it is reasonable to consider the position of RG leaders as uniformly distributed in the area assigned to the AP and evaluate the energy consumed per unit time in transmitting to a RG leader as $\bar{E}_{(a) R G}=\bar{n}_{R G} B_{U} \bar{E}_{A P \rightsquigarrow R G}$, where $\bar{n}_{R G}$ is the average number of reachable users within a RG, $B_{U}$ is the bandwidth for the unicast traffic and $\bar{E}_{A P \rightsquigarrow R G}$ is the average energy per unit area spent to transmit one bit from the AP to the RG leader, which can be evaluated as:

$$
\bar{E}_{A P \rightsquigarrow R G}=\int_{0}^{\min \left(\bar{r}_{A P}, r_{J}\right)} \frac{2 x \rho p_{L} \mathcal{E}(x)}{\bar{r}_{A P}^{2}} \mathrm{~d} x,
$$

where the $\min (\cdot)$ accounts for the fact that when $r_{J}<\bar{r}_{A P}$ the AP serving area $\left(\bar{r}_{A P}\right)$ can not be completely covered by the access point $\left(r_{J}\right)$ and therefore the farthest RG leader reachable by the AP is placed $\min \left(\bar{r}_{A P}, r_{J}\right)$ meters apart from the AP; $\mathcal{E}(x)$ is a mapping giving the minimum energy that can be used to communicate with a node placed $x$ meters apart, i.e.:

$$
\mathcal{E}(x)=\min _{1 \leq j \leq J}\left\{\left(E_{j}^{t x}+E_{j}^{r x}\right) \text { such that } r_{j} \geq x\right\} .
$$

Finally, the average number of unconnected nodes might be found by repeating the approach of section 6-A by replacing

\begin{tabular}{|l|r|r|}
\hline Scenario $\rightarrow$ & \multicolumn{1}{|c|}{ Indoor } & Outdoor \\
\hline Node prob. of having interface 1 & 0.8 & 0.667 \\
Node prob. of having interface 2 & 0.8 & 0 \\
Node prob. of having interface 3 & 0.8 & 0.5 \\
Node prob. of having interface 4 & 0 & 0.9 \\
AP Density $\left(\rho_{A P}\right)$ & $5 \cdot 10^{-5}$ & $4 \cdot 10^{-6}$ \\
\hline
\end{tabular}

Table 1

Parameters of the Scenarios

\begin{tabular}{|l|c|c|c|c|}
\hline Interface $\rightarrow$ & 1 & 2 & 3 & 4 \\
\hline Energy in TX $\left(E^{t x}\right.$, norm. $)$ & 1 & 1.5 & 2 & 10 \\
Energy in RX $\left(E^{r x}\right.$, norm. $)$ & 0.5 & 0.75 & 1 & 2.5 \\
Average coverage radius $(r$, in m) & 10 & 20 & 40 & 130 \\
Period of HELLOs (norm. to $\Delta T)$ & 3 & 3 & 5 & 10 \\
\hline
\end{tabular}

Table 2

Parameters of the Radio Interfaces

$r_{A P}$ with $r_{R G}$, so that one obtains the number $\bar{n}_{j, h}$ of nodes belonging to the $j$ th region of the RG which can be covered by the RG leader by using technology $h$. Summing these values over all regions and all technologies we obtain the number of users reachable by multi-hop through the RG leader, but conditioned to the situation where the RG leader is connected to the AP. However, it is also necessary to account for the cases where the missing coverage is due to lack of connection between the RG leader and an AP, i.e., no AP is present within $r_{J}$ meters from the RG leader. This occurs with probability $\mathcal{P}\left(0, \pi r_{J}^{2}\right)=\exp \left(-\rho_{A P} \pi r_{J}^{2}\right)$. Thus, $\bar{n}_{u}$ is found as:

$$
\bar{n}_{u}=\pi r_{R G}^{2} \rho-\left(1-\exp \left(-\rho_{A P} \pi r_{J}^{2}\right)\right) \sum_{j=1}^{J} \sum_{h=1}^{J} \bar{n}_{j, h} .
$$

\section{RESUlts}

In this section we report some results for two different network cases, whose data are summarized in Table 1 . We consider two possible scenarios with different radio technologies. The characteristics of each radio technology are shown in Table 2 . For both scenarios and all technologies, we also assume that the length of all HELLO messages is 30 bytes and their periods are normalized to $\Delta T$ equal to $1 \mathrm{~s}$.

The indoor scenario might be regarded as a wireless HotSpot, where users are equipped with different short-range technologies, whereas the outdoor scenario might be seen as a network with larger cells, where almost all users have an interface with high range but also several users own additional short-range interfaces in order to extend coverage. The chosen values of $\rho_{A P}$ give an average inter-AP distance of $\bar{d}_{A P} \approx 35.4 \mathrm{~m}$ for the indoor and $\bar{d}_{A P}=125 \mathrm{~m}$ for the outdoor case. The energy consumptions are normalized to the expenditure in transmission of interface 1 . Their values are only to validate the analysis. However, note that they respect the principle that the larger the transmission range, the higher the consumption. This simplifies the notation of our analytical approach as the indexing function introduced in Section 6 can be replaced by an identity function.

In the following, we keep $\rho_{A P}$ fixed by investigating the impact on the performance of the node density $\rho$ and of the probability $p_{L}$ of being RG leader. In Figs. 4 and 5, we report the average number of unconnected users per unit area in 


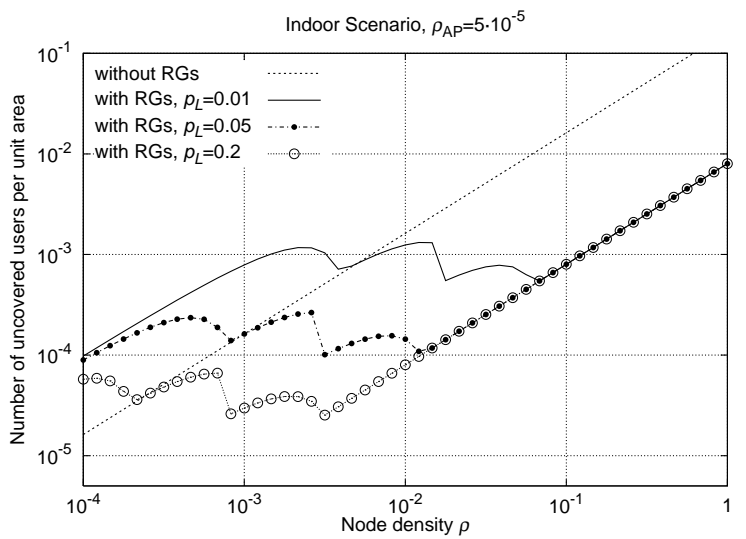

Fig. 4. Average number of uncovered users as a function of the node density $\rho$, indoor scenario.

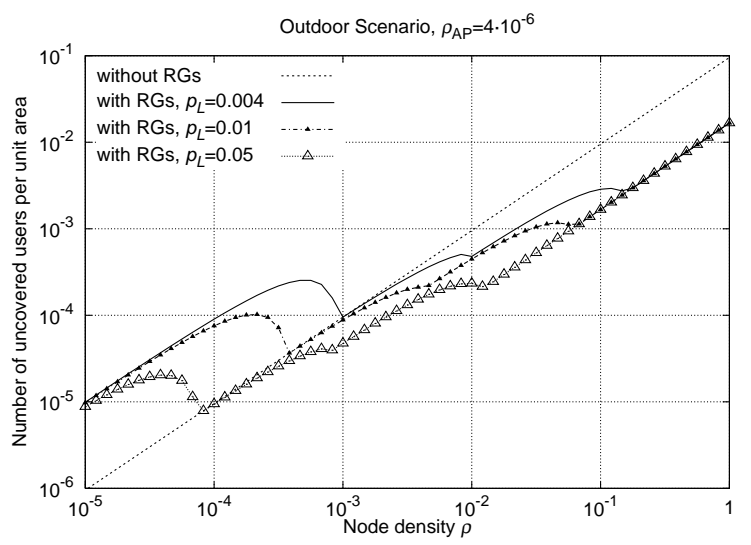

Fig. 5. Average number of uncovered users as a function of the node density $\rho$, outdoor scenario.

both scenarios. These results, as well as the ones shown in the following, have been verified also through simulations, which exhibit good agreement, even though at a preliminary level (see next section). These are the users that, on average and for the given system parameters, can not be reached by any technology and are therefore disconnected from the network. Note that, with our choices of the parameters, it is always possible that some users do not own any interface, as it was discussed in Section 3. More specifically, this occurs with probability $\prod_{j=1}^{J}\left(1-p_{j}\right)$, which is close to $1 \%$ for the considered scenarios. The figures show the case without RGs and the cases where RGs are established for different choices of the parameter $p_{L}$, i.e., of the average size of the groups.

The trend of the case without RGs is always linear, since it only depends on the direct connectivity of the nodes. The RG structures are clearly inefficient when the node density is quite low: there is, in fact, an increase of the probability of being uncovered due to the fact that the RG leader is likely not to be covered by any AP. It might be in fact observed that this holds as long as $\rho p_{L}<\rho_{A P}$, which is a situation where the introduction of RGs is inadequate, since it would force the transmission to wider range than the direct transmission from the APs.

When the node density increases, we observe a descent in the number of uncovered nodes, which occurs in three phases. This is motivated by the fact that three interfaces are avail-

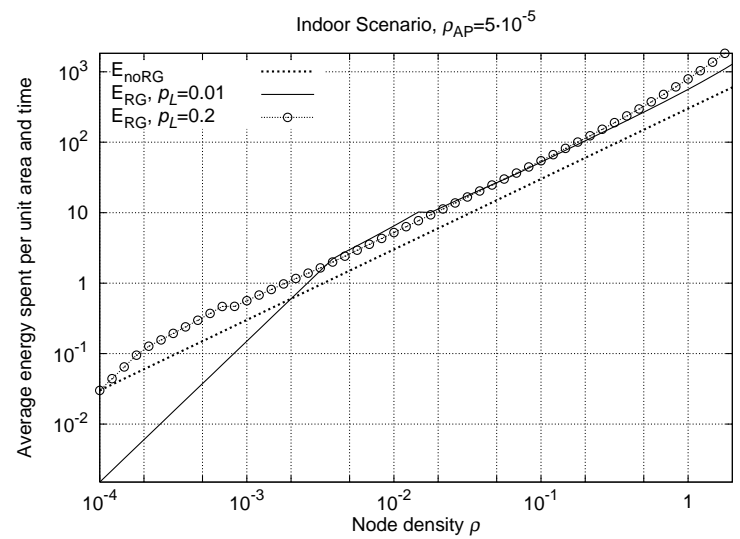

Fig. 6. Total average energy spent per unit area and time, as a function of the node density $\rho$, indoor scenario.

able: roughly speaking, each point of descent corresponds to the additional reachability introduced by a multi-hop routing through the RG leader, by means of a radio interface which is not covered by any AP. The observed behavior is henceforth due to the separation of the coverage radii of the technologies. Note, in fact, that the reduction in the number of uncovered users is less pronounced in the outdoor scenario, where the available radio interfaces provide a wider coverage. In this case, technology diversity appears to be less useful as terminals are almost always reachable through the longest range technology. Of course, this holds here as we do not consider practical aspects such as congestion at the APs and we do not investigate load balancing issues. Moreover, the decrease of the curves in Figs. 4 and 5 occurs for a lower value of $\rho$ as $p_{L}$ increases. This is according to the intuition that, for what concerns the coverage aspect, smaller RGs (higher $p_{L}$ ) perform better, even though we also note that there is a performance floor, corresponding to the case where all users with at least one interface are reached. The aforementioned users without any interface can not of course be reached in any way. Since the parameter $p_{L}$ summarizes the associativity performance of the RG creation algorithm, we infer that by appropriately designing the logical aggregation of nodes one can significantly extend the coverage in the most appropriate manner. In general, we observe that RGs and hence the localized presence of RG leaders (or coordinating/relay entities) are actually good for extending the coverage by therefore substantially reducing to a minimum the probability that a device is disconnected.

In Figs. 6 and 7, we focus on the energy expenditure per unit area and time with and without RGs. For the RG case, the single contributions to the total energy spent, i.e., $\bar{E}_{(a) R G}^{*}$, $\bar{E}_{(b) R G}^{*}$, and $\bar{E}_{(m) R G}^{*}$, are considered in Figs. 8 and 9. We observe that in the considered scenarios the total energy expenditure is generally higher in the RG case, except, of course, when $\rho$ is too low so that several nodes are not covered. The higher energy consumption is due to the fact that the RG leader acts as a relay by first receiving the data from the APs and then re-transmitting to the RG members. This is trivially inefficient from the energy point of view and, for this reason, leads to a higher energy expenditure. Nevertheless, note first of all that in the outdoor case the energy expenditure increase is not that relevant. This is because the local transmissions (RG leader 


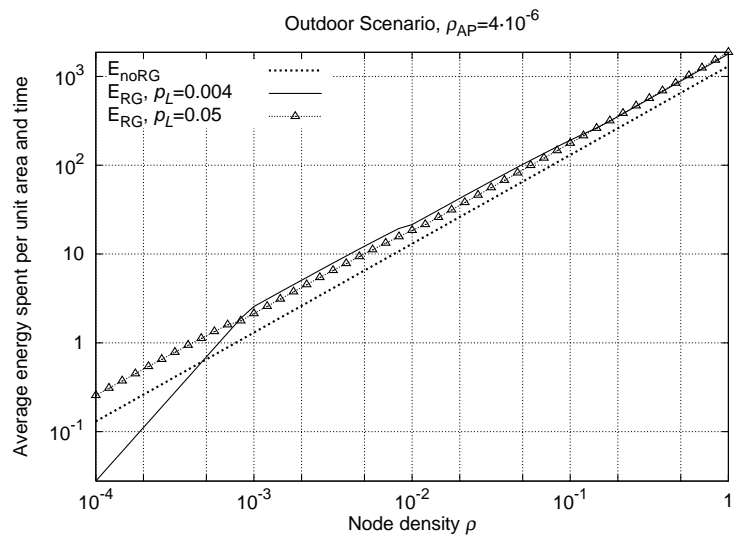

Fig. 7. Total average energy spent per unit area and time, as a function of the node density $\rho$, outdoor scenario.

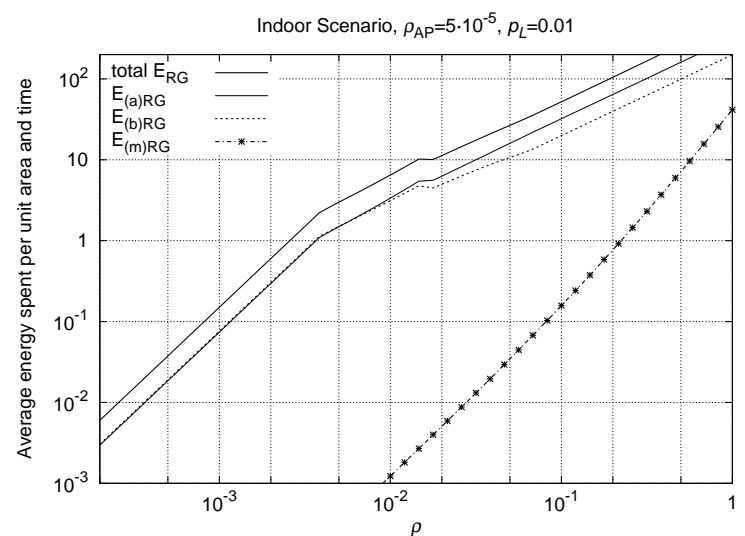

Fig. 8. Total average energy spent per unit area and time in the RG case and its subdivision into its three contributions, as a function of the node density $\rho$, indoor scenario.

$\rightsquigarrow$ nodes) is mostly carried out by means of the low range interfaces 1 and 3, whose energy consumption is the smallest.

Indeed, with a different choice of parameters, which we believe however not to be very realistic, the RGs can also decrease the power consumption (this occurs when the relaying happens through the low-range interfaces, which should be less power demanding). Thus, in certain cases, especially where low-range interfaces with low power expenditure are available, the additional cost of the transmission through RG is not really high. Note in fact that in Fig. 9 the term $\bar{E}_{(b) R G}^{*}$ is significantly lower than $\bar{E}_{(a) R G}^{*}$, whereas in Fig. 8 they were comparable, so that the total power expenditure was more or less doubled (also including the term $\bar{E}_{(m) R G}^{*}$ ).

More in general, we note that the application of the RG concept might lead to save energy for different network scenarios, which can not be addressed here due to the structure of the analytical model but are interesting from the point of view of further research developments beyond the present paper. One possibility is the introduction of the Power Control at the transmitter's side. If the power levels at the transmitter are adjustable, and not fixed as we considered here for the sake of simplicity, we can tune the coverage radii to the desired size. Under this condition, relaying through intermediate nodes might even be energy-saving, since the channel attenuation increases more than linearly with the distance, provided

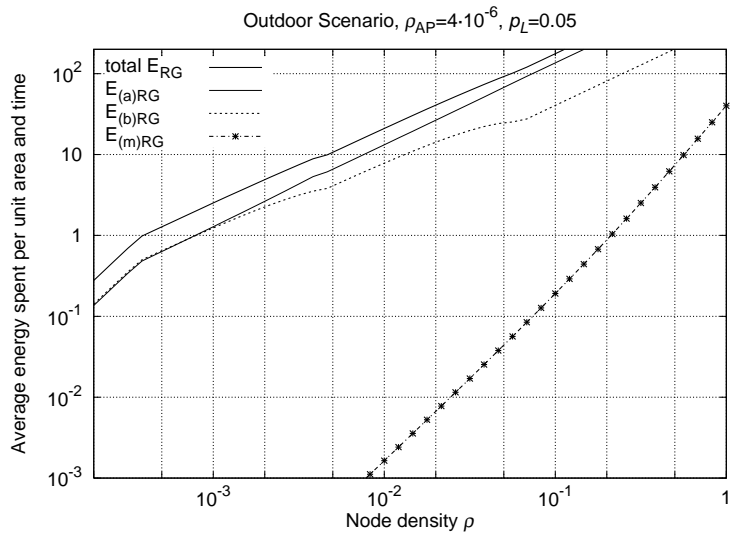

Fig. 9. Total average energy spent per unit area and time in the RG case and its subdivision into its three contributions, as a function of the node density $\rho$, outdoor scenario.

that the angle between the destination and the relay satisfies certain constraints [21]. Another case where the presence of RGs causes energy saving is when the transmission is multicast [21], or in general everywhere the information to deliver can by its own nature be merged through data fusion in packets valid for all destinations, thus leading to counting the term $\bar{E}_{(a) R G}^{*}$ only once for the whole multicast group. In this case, grouping and hence relaying packets is expected to lead to considerable benefits in terms of energy. However, many intricacies appear, as the multicast routing problem is known to be $n p$-hard. Therefore, this topic is left for future research and is not quantitatively addressed here.

Figs. 8 and 9 are worth of more emphasis for what concerns $\bar{E}_{(m) R G}^{*}$. This term is increasing with $\rho$, so that for low node density it is significantly lower than the energy needed to transmit to the end users, but for higher values of $\rho$ this contribution might no longer be negligible. It shall be observed that this term also heavily depends on the vector $\mathbf{E}^{r x}$ and on the HELLO messages transmission periods. Therefore, all RG maintenance parameters must be carefully considered for values of the node density from moderate to high as their impact on the overall energy balance might be relevant.

To sum up, it is possible to say that the creation of RGs introduces a higher energy consumption but provides a higher connectivity that, for reasonable values of $\rho$, exceeds the connectivity of the normal operational mode (AP $\rightsquigarrow$ users) by about one order of magnitude. This is achieved by paying more in terms of energy spent, for both routing and establishing/maintaining RGs. This introduces a trade-off which can be clarified by looking at Figs. 10 and 11 .

Here, the energy consumption is plotted versus the node probability of being disconnected from the network. Thus, suitable solutions are close to the bottom left part of the graph. The curves are obtained for different values of $\rho$, which is a value known a priori and not tunable. Instead, the value $p_{L}$ describes one degree of freedom in the design of the routing group formation strategies, since it directly determines the number of nodes in the same RG. Note that from a general point of view it seems that by increasing $p_{L}$, and henceforth decreasing the RG size, one improves the performance. As an example, for a given power consumption level, the curve with $p_{L}=0.2$ (Fig. 10) leads to a smaller probability of being 


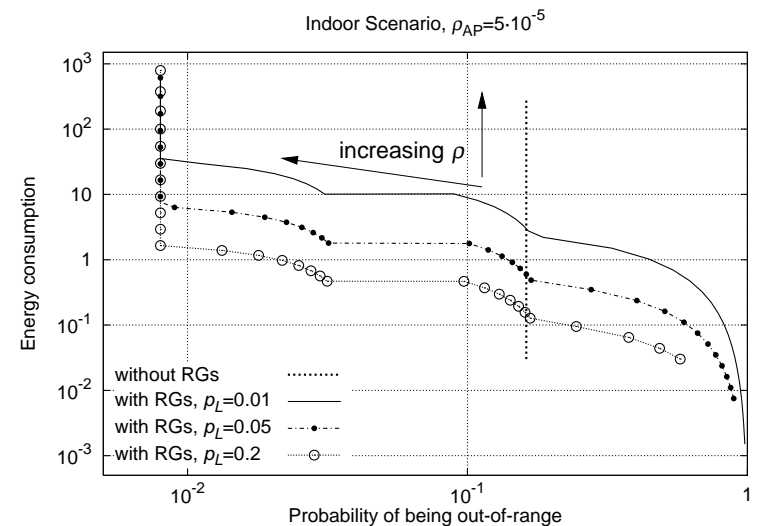

Fig. 10. Tradeoff of the RG formation between energy expenditure and increased coverage, indoor scenario.

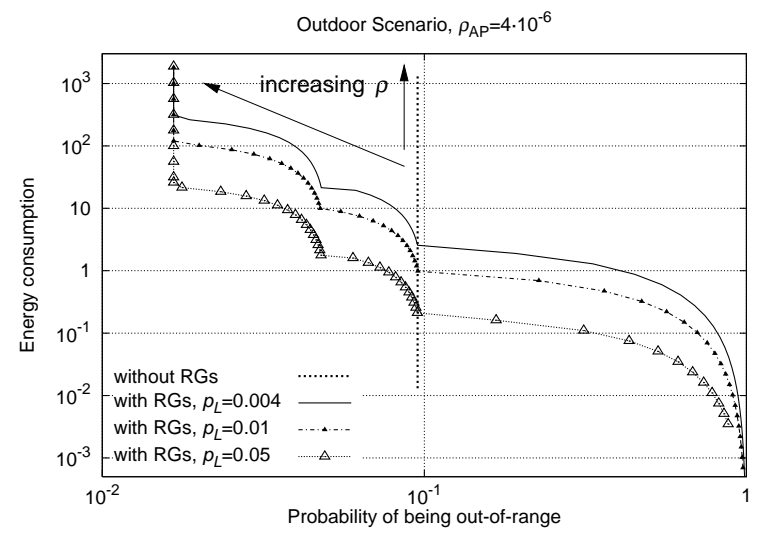

Fig. 11. Tradeoff of the RG formation between energy expenditure and increased coverage, outdoor scenario.

out of range than the case where $p_{L}=0.05$. However, one has to consider the following two facts: the first one is that, in practice, small routing groups are possible only if enough nodes own all access technologies (remember that in our analysis we assumed full availability of the interfaces for the RG leader). Secondly, it is also to be observed that for the same value of $\rho$ the energy expenditure of the smaller RG is higher. This is visible in Fig. 10 from the fact that all points of the curve with $p_{L}=0.2$ are indeed higher than the corresponding points (i.e., those with the same value of $\rho$ ) of the curve with $p_{L}=0.05$.

From a practical point of view, this means that the routing group size is another critical parameter and has to be accurately selected. In fact, when the node density is high, smaller RGs may be preferable: the figures show for example that the cases with RGs and sufficiently high $p_{L}$ obtain for high $\rho$ values comparable energy consumption and substantially better connectivity than the case without RGs (vertical dotted line). When the node density increases further, using small RGs is less advantageous, since it only leads to higher power consumption. In this case, a lower $p_{L}$ is preferable (see the points in the leftmost part of the plots).

As a final comment, note that the tradeoff investigated in Figs. 10 and 11 involves a generic RG formation algorithm, without any optimality criterion about the choice of the AP. We therefore expect an even better performance for realistic

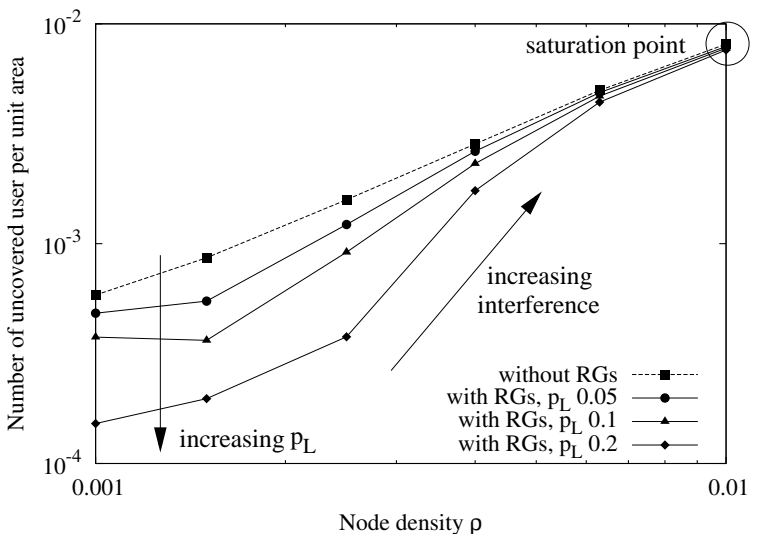

Fig. 12. Simulation results: average number of uncovered users per unit area as a function of the node density $\rho$.

RG formation algorithms, where the leader is not selected randomly but in a more efficient way.

\section{Validation of the Analysis through Sim- ULATION}

In this section, we present accurate simulation results aimed at validating and further investigating some of the above facts. To this end, we used an event-driven network simulator for heterogeneous wireless systems which has been developed within the Ambient Networks project [1]. The channel is modeled accounting for both path loss (Hata model) and multipath fading, which is tracked by means of a Jakes simulator, see [15]. We consider a network scenario composed by two radio access technologies: IEEE802.11b and UMTS. User devices move within a simulation area of $160 \times 160 \mathrm{~m}^{2}$, with speeds uniformly distributed in the range $[0.5,2] \mathrm{m} / \mathrm{s}$, so as to mimic a typical pedestrian scenario. The density $\rho$ of the mobile nodes spans in $[0.001,0.01]$. Mobility patterns are generated according to a random way point mobility model. We consider a single AP, placed at the center of the simulation area and owning both technologies. Exactly $20 \%$ of the mobile devices own both wireless technologies, whereas the remaining $80 \%$ of the population picks one of the two radio technologies at random at the beginning of the simulation. We consider an uplink data transmission. As above, we consider two different access strategies: with and without RGs. In the former case (RGs), each user can access the AP only relaying its data to an in range $\mathrm{RG}$ leader. $\mathrm{RG}$ leaders are elected at random at the beginning of the simulation with probability $p_{L}$ and among the users having both technologies. In the latter case (no RG), relaying is not permitted and a mobile device is connected to the AP if and only if the AP is directly reachable through at least one of the radio technologies owned by the user. Finally, the UMTS network covers the whole simulation area, whereas the IEEE802.11b technology provides a good connectivity up to a distance of approximately $80 \mathrm{~m}$ from the AP. All users generate uplink traffic (users $\rightsquigarrow$ AP) at the rate of one packet per second. Packets are 512 bytes long. Users' traffic is exploited, in part, for the establishment and maintenance of the routes to get to the AP. To this end, we use the DSR protocol modified in such a way that only RG leaders and APs relay data traffic. 
In Fig. 12 we report the density of unconnected users for both scenarios (with and without RGs). As expected, and in accordance with the results discussed in previous sections, the case without RGs gives the worst performance in terms of connectivity. For $\rho \approx 0.001$ the gains offered by RGs increase with $p_{L}$ (the number of RG leaders in the area) and are of about one order of magnitude for $p_{L}=0.2$, i.e., when the number of RG leaders is (on average) $4 \%$ the population size. However, as $\rho$ increases the performance of the RG case saturates to the scenario without RGs. This is basically due to the following two facts: 1) the capacity of the AP is limited 2) an increasing $\rho$ leads to an increasing user interference that, in turn, limits the maximum number of communicating users that can be supported by the system simultaneously. Observe that the point where the performance saturates to the "no RG" curve (saturation point if the figure) can be shifted to the right by exploiting a radio technology which offers a larger capacity or, alternatively, increasing the number of APs in the area. In any event, from Fig. 12 we have a further confirmation of the advantages offered by the RG paradigm which, if correctly exploited, can lead to substantial performance improvements through relaying and cooperation.

\section{Conclusions And Future Work}

In this paper we focused on next generation wireless network scenarios where both users and access points own multiple radio technologies and can therefore communicate exploiting radio technology diversity. In this context, we introduced the concept of routing group (RG) formation as a tool to logically merge users in close proximity and/or moving together. Given the RG concept, we first formulated an analytical framework in order to model the multi-radio scenario, by considering uniform and random user placement and a probabilistic radio interface assignment. Subsequently, we investigated the effectiveness of the user aggregation (RG) approach in terms of energy consumption and connectivity, that we expressed here as the density of unconnected users. We found that, under reasonable assumptions, the RG approach has the potential of dramatically increasing the connectivity metric and, if properly dimensioned, this happens without increasing too much the energy expenditure. Future research is devoted to the design of algorithms for the creation and maintenance of RG structures and their exploitation in routing, topology control and path discovery schemes.

\section{ACKNOWLEDgment}

This work has been carried out in the framework of the Ambient Networks project that is partially funded by the Commission of the European Union. The views expressed in this paper are solely those of the authors and should not be interpreted as necessarily representing the views of their employers, this project or the European Commission. We are most grateful to Marco Miozzo and Nicola Bui for their support with the simulator used to derive the results in Section 8.

\section{REFERENCES}

$$
\begin{aligned}
& \text { [1] "The Ambient } \quad \text { Networks } \\
& \text { http://www.ambient-networks.org. }
\end{aligned}
$$

[2] J. Sachs, H. Wiemann, J. Lundsjö, and P. Magnusson, "Integration of multi-radio access in a beyond 3G network," in Proceedings of IEEE PIMRC, 2004, pp. 757-762.

[3] Q. Huang, C. Julien, and G.-C. Roman, "Relying on safe distance to achieve strong partitionable group membership in ad hoc networks," IEEE Transactions on Mobile Computing, vol. 3, no. 2, pp. 192-205, 2004.

[4] P. Mohapatra, C. Gui, and J. Li, "Group communications in mobile ad hoc networks," IEEE Computer Magazine, vol. 37, no. 2, pp. 52-59, 2004.

[5] G.-C. Roman, Q. Huang, and A. Hazemi, "Consistent group membership in ad hoc networks," in Proceedings of ICSE, 2001, pp. 381-388.

[6] Y.-L. Chang and C.-C. Hsu, "Connection-oriented routing in ad hoc networks based on dynamic group infrastructure," in Proceedings of ISCC, 2000, pp. 587-592.

[7] M. Zonoozi and P. Dassanayake, "User mobility modeling and characterization of mobility patterns," IEEE Journal on Selected Areas in Communications, vol. 15, pp. 1239-1252, 1997.

[8] C. Toh, "Associativity-based routing for ad-hoc mobile networks," Kluwer Wireless Personal Communications, vol. 4, pp. 103-139, 1997.

[9] D. Stoyan, W. S. Kendall, and J. Mecke, Stochastic Geometry and its Applications, 2nd ed. John Wiley \& Sons, 1995.

[10] D. Dardari, A. Conti, and R. Verdone, "Process estimation through self-organizing collaborative wireless sensor networks," in Proceedings of IEEE Globecom, vol. 5, 2004, pp. 3193-3199.

[11] S. Basagni, "Distributed and mobility-adaptive clustering for multimedia support in multi-hop wireless networks," in Proceedings of IEEE VTC 1999 Fall, vol. 2, 1999, pp. 889-893.

[12] M. Chatterjee, S. Das, and D. Turgut, "WCA: a weighted clustering algorithm for mobile ad hoc networks," Kluwer Cluster Computing, vol. 5, pp. 193-204, 2002.

[13] H. Thompson, "Distribution of Distance to Nth Neighbour in a Population of Randomly Distributed Individuals," Ecology, vol. 37, no. 2, pp. 391-394, apr 1956.

[14] R. J. Kryscio and R. Saunders, "On interpoint distances for planar Poisson cluster process," Journal of Applied Probability, vol. 20, pp. 513-528, 1983.

[15] G. Stüber, Principles of Wireless Communications, 2nd ed. Kluwer Academic, 1896.

[16] M. Månsson, "Poisson approximation in connection with clustering of random points," Annals of Applied Probability, vol. 9, pp. 465-492, 1999.

[17] B. McDonald and T. Znati, "Comparative analysis of neighbor greeting protocols: ARP versus ES-IS," in Proceedings of IEEE SIMULATION' 96, 1996, pp. 71-80.

[18] C. Prehofer and B. Souville, "Synchronized reconfiguration of a group of mobile nodes in ad-hoc networks," in Proceedings of IEEE ICT 2003, vol. 1, 2003, pp. 400-405.

[19] M. Rossi, N. Bui, L. Badia, and M. Zorzi, "On Group Mobility Patterns and their Exploitation to Logically Aggregate Terminals in Wireless Networks," in Proceedings of IEEE VTC Fall, Dallas, Texas, US, Sep 2005.

[20] B. A. Miller and C. Bisdikian, Bluetooth revealed: The Insider's Guide to an Open Specification for Global Wireless Communications. Prentice Hall, 2000.

[21] J. Wieselthier, G. Nguyen, and A. Ephremides, "Multicasting in energy-limited ad-hoc wireless networks," in Proceedings of IEEE MILCOM 1998, vol. 3, 1998, pp. 723-729.

\section{Authors' Biographies}

Michele Rossi (rossi@ dei.unipd.it) received a Laurea Degree in Electrical Engineering (with honors) and a Ph.D. in Information Engineering from the University of Ferrara in 2000 and 2004, respectively. From March 2000 to November 2005 he was a research fellow in the Department of Engineering at the University of Ferrara. During 2003, he was on leave at the Center for Wireless Communications (CWC) at the University of California, San Diego (UCSD), where he did research on wireless sensor networks. In November 2005, he joined 
the Department of Information Engineering at the University of Padova, Italy, where he is currently an assistant professor. Dr. Rossi has been working as principal investigator in several National and EU funded research projects. His current research interests are mainly focused on access selection algorithms for heterogeneous wireless networks and protocols for wireless sensor networks, including network reprogramming techniques, network coding enhanced data gathering and correlation aware MAC schemes.

Leonardo Badia (1.badia@imtlucca.it) was born in Ferrara, Italy, in 1977. He received the Laurea Degree (with honors) in Electrical Engineering and the Ph.D. in Information Engineering from the University of Ferrara, Italy, in 2000 and 2004, respectively. During 2002 and 2003 he was on leave at the Radio System Technology Labs (now Wireless@KTH), Royal Institute of Technology of Stockholm, Sweden. After having been with the Engineering Department of the Università di Ferrara, Italy, he joined in 2006 the "Institutions Markets Technologies" (IMT) Lucca Institute for Advanced Studies, in Lucca, Italy, where he is currently a Research Fellow. He also collaborates with DEI, University of Padova, Italy. His research interests include energy efficient Ad Hoc Networks, transmission protocol modeling, Admission Control and economic modeling of Radio Resource Management for Wireless Networks. Dr. Badia serves also as reviewer for several periodicals in the communication area.

Paolo Giacon (paolo.giacon@unipd.it) was born in Camposampiero (Padova), Italy, in 1979. In 2004 he received the Laurea degree in telecommunication engineering from the University of Padova with a thesis on hybrid radio devices. From december 2004 to end of 2005 he was with the Consorzio Nazionale Interuniversitario per le Telecomunicazioni (CNIT) at the Research Unit of Ferrara (University of Ferrara), where he did research on protocols for multi-radio wireless terminals and multimedia communications. Since January 2006 he has been a PhD student at the Department of Innovation in Mechanics and Management of the University of Padova, Italy.
Michele Zorzi (zorzi@ dei.unipd.it) received a Laurea degree and a Ph.D. in electrical engineering from the University of Padova, Italy, in 1990 and 1994, respectively. During academic year 1992/1993, he was on leave at the University of California, San Diego (UCSD), attending graduate courses and doing research on multiple access in mobile radio networks. In 1993 he joined the faculty of the Dipartimento di Elettronica e Informazione, Politecnico di Milano, Italy. After spending three years with the Center for Wireless Communications at UCSD, in 1998 he joined the School of Engineering of the University of Ferrara, Italy, where he became a professor in 2000. Since November 2003 he has been on the faculty at the Information Engineering Department of the University of Padova. His present research interests include performance evaluation in mobile communications systems, random access in mobile radio networks, ad hoc and sensor networks, and energy constrained communications protocols. $\mathrm{He}$ is the Editor-In-Chief of IEEE Wireless Communications, and currently serves on the Editorial Boards of IEEE Transactions on Communications, IEEE Transactions on Wireless Communications, IEEE Transactions on Mobile Computing, Wiley Journal of Wireless Communications and Mobile Computing, and the ACM/URSI/Kluwer Journal of Wireless Networks. He was also guest editor for special issues in IEEE Personal Communications (Energy Management in Personal Communications Systems) and IEEE Journal on Selected Areas in Communications (Multimedia Network Radios). 